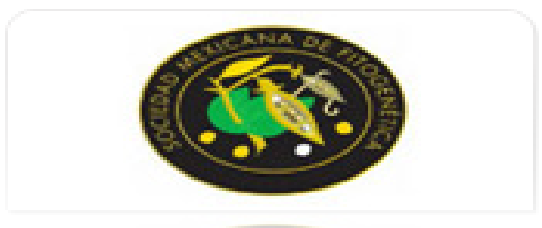

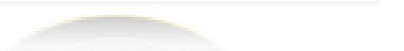

\section{Revista Fitotecnia Mexicana}

ISSN: 0187-7380

revfitotecniamex@gmail.com

Sociedad Mexicana de Fitogenética, A.C.

México

Espinosa-Pérez, Esteli N.; Ramírez-Vallejo, Porfirio; Crosby-Galván, M. Magdalena;

Estrada-Gómez, J. Arturo; Lucas-Florentino, Bernardo; Chávez-Servia, José L.

CLASIFICACIÓN DE POBLACIONES NATIVAS DE FRIJOL COMÚN DEL CENTRO-SUR

DE MÉXICO POR MORFOLOGÍA DE SEMILLA

Revista Fitotecnia Mexicana, vol. 38, núm. 1, 2015, pp. 29-38

Sociedad Mexicana de Fitogenética, A.C.

Chapingo, México

Disponible en: http://www.redalyc.org/articulo.oa?id=61035375005

Cómo citar el artículo

- Número completo

- Más información del artículo

- Página de la revista en redalyc.org

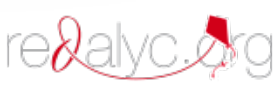

Sistema de Información Científica

Red de Revistas Científicas de América Latina, el Caribe, España y Portugal Proyecto académico sin fines de lucro, desarrollado bajo la iniciativa de acceso abierto 


\title{
CLASIFICACIÓN DE POBLACIONES NATIVAS DE FRIJOL COMÚN DEL CENTRO-SUR DE MÉXICO POR MORFOLOGÍA DE SEMILLA
}

\section{CLASSIFICATION OF COMMON DRY BEAN LANDRACES FROM THE SOUTH-CENTER OF MÉXICO BY SEED MORPHOLOGY}

\author{
Esteli N. Espinosa-Pérez ${ }^{1}$, Porfirio Ramírez-Vallejo ${ }^{1 \dagger}$, M. Magdalena Crosby-Galván ${ }^{1}$, \\ J. Arturo Estrada-Gómez ${ }^{1}$, Bernardo Lucas-Florentino ${ }^{2}$ y José L. Chávez-Servia ${ }^{3 \star}$
}

\begin{abstract}
${ }^{1}$ Postgrado en Recursos Genéticos y Productividad-Genética, Campus Montecillo, Colegio de Postgraduados. Km 36.5 Carr. México-Texcoco. 56230, Montecillo, Texcoco, Edo. de México. ${ }^{2}$ Depto. de Toxicología y Nutrición, Facultad de Química, Universidad Nacional Autónoma de México. 04510. México, D. F. ${ }^{3}$ CIIDIR Unidad Oaxaca, Instituto Politécnico Nacional. Hornos No. 1003. 71230, Santa Cruz Xoxocotlán, Oaxaca.
\end{abstract}

*Autor para correspondencia (jchavezs@ipn.mx)

\section{RESUMEN}

Se evaluó la morfología de semillas de 75 poblaciones de frijol común (Phaseolus vulgaris L.) de hábito indeterminado (tipo IV), colectadas en los estados de México, Morelos, Oaxaca, Guerrero y Tlaxcala. Se obtuvo información relacionada con la zona geográfica, nombre del agricultor, localidad, municipio y estado; así como aspectos de manejo agronómico y de semillas. Se realizaron análisis descriptivos y clasificatorios de componentes principales y de agrupamiento jerárquico con base en la distancia euclidiana, respectivamente, con base en seis características de semilla. Se clasificaron 13 grupos de diversidad de colores y nombres locales usados por los agricultores para distinguir las poblaciones de frijol, donde los más frecuentes fueron: amarillo, ensaladilla, rojo, negro y vaquita. En el análisis de componentes principales, las variables de mayor valor discriminatorio de la variación total, entre poblaciones, fueron: longitud, ancho, peso y volumen de semillas y peso específico. En el análisis de conglomerados se determinaron siete grupos fenotípicos: dos grupos del Estado de México, uno de semilla grande y otro de semilla mediana; dos grupos de semillas pequeñas, uno de Guerrero y otro de Oaxaca; y tres complejos grupales, uno con poblaciones de Tlaxcala y Oaxaca, otro con poblaciones de Oaxaca y Guerrero, y uno más que agrupó a poblaciones de Oaxaca, Guerrero, Estado de México, Morelos y Tlaxcala. Las características de semilla permitieron describir y clasificar la diversidad fenotípica de frijol común en el centro-sur de México.

Palabras clave: Phaseolus vulgaris, color de semilla, morfología, poblaciones nativas.

\section{SUMMARY}

Seed morphology of 75 common bean (Phaseolus vulgaris L.) populations from indeterminate habit (type IV) were evaluated. Seeds were collected in the States of México, Morelos, Oaxaca, Guerrero and Tlaxcala. Data of geographic zone, farmer' name, locality, municipality, state, agronomical and seed management techniques, were gathered at collect time. Descriptive and classificatory analysis of principal components and hierarchical clustering based on Euclidian distances were undertaken using seed traits, in order to determine the relationships among populations. There were 13 groups of color and locales names used by farmers to distinguish the bean populations, where the most frequent classes were amarillo, ensaladilla, rojo, negro and vaquita. Length, wide, weight and volume of seeds and specific weight were the major discriminative variables with respect to the total variation, ac- cording to the principal components analysis. Seven phenotypic groups were determined by cluster analysis: two population groups came from Estado de México, one of big seed size and other medium one. Two groups of small seed were collected in Guerrero and Oaxaca; and three group complexes, the first included populations of Tlaxcala and Oaxaca, other integrated with populations from Oaxaca and Guerrero, and the third one grouped populations of Oaxaca, Guerrero, Estado de México, Morelos and Tlaxcala. Therefore, the seed traits supported the description and classification of the phenotypic diversity of common dry bean from the south-center of México.

Index words: Phaseolus vulgaris, seed color, morphology, landraces.

\section{INTRODUCCIÓN}

En un periodo de 7000 a 8000 años con la influencia de las fuerzas evolutivas de mutación, migración, deriva genética y selección empírica practicada por el hombre, el frijol común (Phaseolus vulgaris L.) evolucionó desde la forma silvestre de hábito trepador y semillas pequeñas distribuida en las tierras altas de Mesoamérica y Zona Andina, a la forma actual que se siembra en diversos ambientes y sistemas de producción tradicional y modernos, lo que produjo cambios morfológicos, fisiológicos y genéticos en este cultivo. En los aspectos morfológicos, el hombre centró su atención en semillas de mayor tamaño y de colores más atractivos, vainas más grandes y menos dehiscentes; adicionalmente, ocurrió la aparición de formas arbustivas y el moteado de la testa en la semilla (Lépiz et al., 2010).

El frijol común tiene una gran tradición que se remonta a tiempos prehispánicos, y presenta amplia diversidad de formas silvestres y cultivadas. Si bien la mayoría de estas formas se localiza a lo largo de la Sierra Occidental entre 500 y $1800 \mathrm{~m}$ de altitud, su cultivo se realiza extensivamente en casi todas las condiciones agroecológicas del país y sistemas de producción (Vidal-Barahona et al., 2006). 
En la actualidad los agrosistemas tradicionales de producción, generalmente de secano (temporal) y en ciertos casos de policultivo, prevalecen en los Valles Altos de México (más de $2000 \mathrm{~m}$ de altitud) y en otras regiones aledañas con altitudes inferiores del centro-sur del país. Aunque la extensión ocupada con estos sistemas se desconoce con precisión, se estima en seis millones de hectáreas y en dos millones el número de familias campesinas ocupadas en esta actividad (Bellon et al., 2009).

A pesar de la importancia ecológica y económica de estos agrosistemas, la información acerca del valor agronómico y bromatológico de las poblaciones de frijol común, así como la relativa al grado de diversidad intra e inter específica en las regiones en que se cultivan, es escasa, dispersa y poco sistematizada. Además, los programas de investigación dirigidos a su aprovechamiento en forma directa o como fuentes de genes, son limitados. Los cambios socioeconómicos y culturales ocurridos en las regiones donde se cultiva este germoplasma ponen en riesgo de pérdida este material biológico y sus agrosistemas de producción asociados.

Esta situación resalta la necesidad de conocer la variación y diversidad, tanto genética como morfológica, para definir estrategias de rescate, conservación y aprovechamiento de poblaciones autóctonas de hábito indeterminado (tipo IV), cultivadas en asociación con maíz (Zea mays L.) (Nadal, 2000; Salinas et al., 2008). La asociación maíz-frijol es más eficiente productivamente por unidad de superficie que los monocultivos, favorece y conserva in situ la diversidad genética de las especies, mantiene la dinámica evolutiva de especies y poblaciones, hay interacciones simbióticas o de cooperación para fijar nitrógeno, favorece el manejo de plagas y enfermedades, y es un espacio de aprendizaje y transmisión de conocimiento local de padres a hijos en los diversos grupos socioculturales (Vizcarra y Marín, 2006; Aguilar et al., 2007; Pinedo et al., 2009).

Entre los factores que han incidido en este fenómeno se encuentran la sustitución de poblaciones nativas por variedades mejoradas, fenómenos meteorológicos, cambios en los sistemas de producción y uso de la tierra, y abandono de tierras o de la actividad agrícola. Estos fenómenos remarcan la necesidad de realizar un proceso continuo de conservación, caracterización morfológica y agronómica de germoplasma, así como de análisis de la distribución geográfica de las poblaciones nativas, ante la posible pérdida irreparable de este germoplasma (Bellon y Berthaud, 2006).

La producción en los agrosistemas tradicionales se basan fundamentalmente en el uso de poblaciones nativas que se caracterizan por presentar amplia diversidad en morfotipos de planta, frutos y semillas; tolerancia a plagas y enfermedades (coevolución); adaptación a condiciones edáficas y culturales específicas y a formas tradicionales de producción; albergar a un número infinito de fenotipos y genotipos mantenidos por cruzamiento natural; construir un poderoso mecanismo de amortiguamiento de los efectos negativos de los factores abióticos y bióticos; y tener un alto valor regional y local por satisfacer requerimientos específicos (López et al., 2005; Castillo et al., 2006).

En particular, las poblaciones de frijol de hábito indeterminado que se cultivan en asociación con maíz y otras especies, presentan amplia riqueza genética, desarrollada y conservada por generaciones de agricultores, asociada con el conocimiento tradicional, y con potencial de producción en condiciones adecuadas de cultivo (Singh et al., 1991a; López et al., 2005; Vargas-Vázquez et al., 2008; HernándezLópez et al., 2013).

Estas poblaciones regularmente se ubican en áreas con variadas condiciones ambientales de humedad, temperatura y suelo: se cultivan en estaciones de crecimiento largas; presentan diversidad genética, fenotípica, adaptabilidad y rusticidad amplia; y pueden adaptarse a sistemas de producción con especies múltiples. Sin embargo, están sujetas a una erosión genética severa por la sustitución de agrosistemas y la introducción de variedades mejoradas, así como por fenómenos socio-culturales relacionados con la migración y el abandono de tierras (Boege, 2010; Soleri et al., 2013).

Algunas poblaciones tienen un alto valor agregado, ya que se han desarrollado para aprovecharlas en forma de ejote (frutos frescos) (Romero-Arenas et al., 2013). Estas poblaciones han sido poco utilizadas en programas genotécnicos (escaso número de variedades mejoradas), y los estudios sistemáticos orientados a su conservación y aprovechamiento son escasos en México.

La presente investigación se realizó con el objetivo de conocer el grado de variación y diversidad en características morfológicas de semilla de poblaciones de frijol común de hábito indeterminado colectadas en cuatro entidades federativas del centro sur de México.

\section{MATERIALES Y MÉTODOS}

Se evaluaron 75 poblaciones nativas de frijol común (hábito IV) provenientes de cuatro entidades del centro-sur de México (Cuadro 1) que fueron colectadas directamente con agricultores: 45 del Estado de México, cuatro de Morelos (como parte de la misma región ecológica del Estado de México); 10 de Guerrero (área de la Montaña), 15 de Oaxaca (Mixteca y región de Tlaxiaco), y cinco de Tlaxcala (municipio de Españita y Vicente Guerrero). En estas regiones el frijol se cultiva en sistemas tradicionales complejos en 
asociación con maíz o maíz-frijol-calabaza (Cucurbita sp.).

Las variables morfológicas evaluadas en la semilla fueron: longitud $(\mathrm{cm})$, ancho $(\mathrm{cm})$, grosor $(\mathrm{cm})$, peso de 100 semillas $(\mathrm{g})$, volumen de 100 semillas $\left(\mathrm{cm}^{3}\right)$, peso especí- fico (relación peso/volumen de 100 semillas, $\mathrm{g} / \mathrm{cm}^{3}$ ), y en forma visual se evaluó el color de testa. De cada población se tomaron 10 muestras representativas de 100 semillas. La longitud, anchura y grosor se midieron con un vernier, en grupos de 100 semillas y se calculó el promedio. Para

Cuadro 1. Clave de colecta (Col.), origen y nombre común de 75 poblaciones de frijol de tipo indeterminado cultivadas en asociación con maíz en los estados de México (Méx.), Morelos (Mor.), Oaxaca (Oax.), Guerrero (Gro.) y Tlaxcala (Tlax.).

\begin{tabular}{|c|c|c|c|c|c|c|c|}
\hline Col. & Localidad de origen & Entidad & Nombre común & Col. & Localidad de origen & Entidad & Nombre común \\
\hline E1 & Cuecuecuautitla (El Pueblito) & Méx. & Rojo o vaquita & $\mathrm{E} 41$ & Tepetlixpa & Méx. & Rojo \\
\hline E2 & San Andrés Tlalamac & Méx. & Amarillo bola & $\mathrm{E} 42$ & Tepetlixpa & Méx. & Vaquita \\
\hline E3 & San Andrés Tlalamac & Méx. & Flor de mayo & $\mathrm{E} 43$ & San Mateo Tecalco & Méx. & Frijol negro \\
\hline E5 & Cuecuecuautitla (El Pueblito) & Méx. & Revueltito & E44 & Cuecuecuautitla (El Pueblito) & Méx. & Mezcla palacio-amarillo \\
\hline E6 & Atlatlahuacán & Mor. & Colado & $\mathrm{E} 45$ & Cuecuecuautitla (El Pueblito) & Méx. & Alubia o novia \\
\hline E7 & Atlatlahuacán & Mor. & Vaquita & E46 & San Andrés Tlalamac & Méx. & Amarillo ovalado \\
\hline E8 & Nepantla & Méx. & Amarillo & $\mathrm{E} 47$ & San Andrés Tlalamac & Méx. & Amarillo boludo \\
\hline E9 & Nepantla & Méx. & Amarillo & O9 & Chamizal, Ticua & Oax. & Negro ejotero \\
\hline E10 & Nepantla & Méx. & Vaquita & $\mathrm{O} 28$ & Barrio San Sebastián Tlaxiaco & Oax. & Frijol colorado \\
\hline E11 & Tepetlixpa & Méx. & Amarillo bola & $\mathrm{O} 29$ & Santa Lucrecia & Oax. & Frijol tempranero \\
\hline E12 & Totolapa & Mor. & Apetito & $\mathrm{O} 33$ & Santa Catarina Ticua & Oax. & Revuelto \\
\hline E13 & Totolapa & Mor. & Cacahuate & $\mathrm{O} 35$ & Primavera Santiago Yosondua & Oax. & Revuelto \\
\hline E14 & San José Tlacotitlan & Méx. & Amarillo & $\mathrm{O} 36$ & San Martín Huamelulpan & Oax. & Tempranero blanco \\
\hline E15 & Tepetlixpa & Méx. & Vaquita & $\mathrm{O} 38$ & Totonando & Oax. & Tempranero \\
\hline E17 & San Andrés Tlalamac & Méx. & Garrapato & O39 & San Pedro Molinos & Oax. & Frijol de ejote \\
\hline E18 & San Andrés Tlalamac & Méx. & Vaquita & $\mathrm{O} 40$ & Santa María Tataltepec & Oax. & Frijol blandito rojo y negro \\
\hline E19 & Cuecuecuautitla (El Pueblito) & Méx. & Amarillo & $\mathrm{O} 42$ & Santa María Tataltepec & Oax. & Negro o frijol grueso \\
\hline E20 & San Andrés Tlalamac & Méx. & Flor de mayo & $\mathrm{O} 43$ & Santa Catarina Tayata & Oax. & Frijol colorado \\
\hline E21 & Cuecuecuautitla (El Pueblito) & Méx. & Color de rosita & $\mathrm{O} 45$ & Haquiniyiqui & Oax. & Negro ayocote \\
\hline E22 & San AndrésTlalamac & Méx. & Rojo & $\mathrm{O} 46$ & Haquiniyiqui & Oax. & Cuarenteño \\
\hline E23 & Cuijingo & Méx. & Coconita & $\mathrm{O} 49$ & Primavera, Santiago Yosondua & Oax. & Ejote suave \\
\hline E24 & Nepantla & Méx. & Flor de mayo & O51 & San Isidro & Oax. & Amarillo \\
\hline E25 & San Andrés Tlalamac & Méx. & Vaquita & G87 & Almolonga & Gro. & Frijol rojo \\
\hline E26 & San Andrés Tlalamac & Méx. & Torito & G94 & Chilacachapa & Gro. & Pintito de vara \\
\hline E27 & San Andrés Tlalamac & Méx. & Garrapato o morito & G96 & Zoquiapán & Gro. & Frijol negro de vara \\
\hline E28 & San Andrés Tlalamac & Méx. & Colorado & G97 & Zoquiapan & Gro. & Negro de vara o milpa \\
\hline E29 & San Andrés Tlalamac & Méx. & Amarillo & G99 & Santa Ana & Gro. & Frijol rojo de vara \\
\hline E30 & Nepantla & Méx. & Palacio & G103 & El Refugio & Gro. & Frijol rojo de vara \\
\hline E31 & Nepantla & Méx. & Vaca & G118 & Las Trancas & Gro. & Frijol rojo de vara \\
\hline E32 & San José Tlacotitlán & Méx. & Negro bola & G120 & Las Trancas & Gro. & Rojo de cáscara dura \\
\hline E33 & San José Tlacotitlán & Méx. & Media oreja & G129 & Zompepelco & Gro. & Frijol rojo de vara \\
\hline E34 & Atlautla & Méx. & Amarillo & G137 & El Nuevo Paraíso & Gro. & Frijol blanco enredador \\
\hline E35 & Nepantla & Méx. & Palacio & $\mathrm{T} 1$ & Vicente Guerrero & Tlax. & Mantequilla \\
\hline E36 & San Andrés Tlalamac & Méx. & Amarillo & $\mathrm{T} 2$ & Vicente Guerrero & Tlax. & Vaquita \\
\hline E37 & San Andrés Tlalamac & Méx. & Apetito & $\mathrm{T} 3$ & Vicente Guerrero & Tlax. & Moradito o morita \\
\hline E38 & San Lorenzo Tlaltecoyac & Méx. & Negro bola & $\mathrm{T} 4$ & Vicente Guerrero & Tlax. & Mantequilla \\
\hline E39 & Juchitepec & Méx. & Color de rosa & T5 & Vicente Guerrero & Tlax. & Pinto \\
\hline E40 & Cuecuecuautitla (El Pueblito) & Méx. & Sanate o cabeza de ahuate & & & & \\
\hline
\end{tabular}


el volumen se utilizó el método de desplazamiento de agua, en una probeta de vidrio y agua con volumen preestablecido, en el interior de la probeta con agua y volumen conocido se colocaron 100 semillas sanas y sin fisuras en la cubierta, la diferencia originada por las semillas se registró como el volumen (de Allende et al., 2006; Solano-Cervantes et al., 2009).

Para obtener la información base se aplicaron cuestionarios de manera directa a los productores, en sus domicilios o en sus parcelas, en algunos casos en mercados regionales, en los que se registró la ubicación geográfica, nombre del agricultor o colaborador, localidad, municipio y estado. Adicionalmente, cuando fue posible se obtuvo información sobre las condiciones de cultivo, forma de conservación de la población, manejo agronómico y de semillas.

Los análisis estadísticos se hicieron con los programas Infostat (Di-Rienzo et al., 2008) y SAS® (2002). Primero se hizo un análisis descriptivo de frecuencias de color de grano y nombres locales asociados con la distinción de tipos de frijol. Después, a los promedios estandarizados por población se aplicó un análisis de componentes principales (ACP) con base en la matriz de correlaciones, y la dispersión de las poblaciones se graficó sobre el plano determinado por los dos primeros componentes principales. Posteriormente, se hizo un análisis de conglomerados donde el punto de corte se estableció mediante el valor significativo de pseudo $t(P<0.05)$. Por último, a través del análisis de conglomerados se estimaron las relaciones entre características de semilla.

\section{RESULTADOS Y DISCUSIÓN}

Entre las variables destacó la preferencia que se tiene en cada zona geográfica por el color y tamaño de la semilla, lo que se reflejó en la frecuencia de colores y nombres locales utilizados para distinguir los tipos de frijol (Figura 1). En las áreas de colecta se observó que los agricultores conservan la diversidad en la forma de semilla, precocidad y hábitos de crecimiento y su uso en diversos sistemas de producción, que les permiten afrontar con éxito las restricciones ambientales.

Durante los recorridos de campo se observó que la mayoría de agricultores disponían de diferentes parcelas de producción de alimentos básicos, de tal manera que el frijol se encontró inmerso en variados sistemas de producción tradicional; principalmente en asociación con maíz, pero también con otros cultivos hortícolas; por ejemplo, tomate (Solanum lycopersicum L.), pepino (Cucumis sativus L.) y cilantro (Coriandrum sativum L.), estos últimos con espaldera. Estos cambios en los patrones de asociación se atribuyen a que en ocasiones el tallo del maíz no soporta suficientemente al frijol, o porque las poblaciones de frijol empleadas son de crecimiento agresivo. En algunas comunidades de Guerrero y Oaxaca el frijol trepador se cultiva con espalderas, sobre todo para la producción de ejote.

Las poblaciones de frijol colectadas se consideran producto de la conservación in situ realizada por agricultores en comunidades que las han preservado desde hace 30 años o más, frecuentemente por familias de escasos recursos económicos en las que el grano constituye la base alimenticia. Además de conservar los acervos genéticos, el conocimiento local asociado con las prácticas de cultivo, beneficio de grano y criterios de selección de semilla para la siguiente siembra, entre otros aspectos, se transmite de generación en generación. La adaptación de las poblaciones a condiciones restrictivas de producción y a sistemas agrícolas específicos, así como el prolongado proceso de selección a que han sido sometidas, son ejemplos del valor genético que encierran estas poblaciones nativas (Singh et al., 1991a; Soleri et al. 2013).

La muestra colectada abarcó 13 colores y nombres locales (Figura 1). Los colores y nombres locales predominantes fueron Amarillo y Ensaladilla (mezcla de tipos y colores de semilla), ambos con una frecuencia de $18.7 \%$, seguido del Rojo (12\%), y luego por Negro y 'Vaquita' (variegado de crema y rojo), con $10.7 \%$ del total. Los siguientes fueron un complejo de varios colores con frecuencia de $5.3 \%$, después Café, Lila, Beige y Flor de mayo con $4 \%$. Los colores menos frecuentes fueron la Alubia (blanco), Garrapato (gris con manchas negras) y Torito a veces llamado Vaquita pinta (crema con matices negros) con $2.7 \%$.

Esta clasificación genérica es la reconocida por el agricultor y es la manera en que manejan su germoplasma. Solano-Cervantes et al. (2009) documentaron que el frijol de colores negro y rojo predomina en el mercado regional de Tlatzalapa, Guerrero, debido a la fuerte demanda local. Según Rocandio et al. (2009), en los mercados regionales de Ozumba y Chalco, Estado de México, los tipos más frecuentes fueron el Amarillo, Ensaladilla, Coconita y Garrapato, que presumiblemente están vinculados con su valor comercial, cultural y culinario. En el presente estudio los tipos más frecuentes también desempeñan una función económica y social alimentaria importante. Por ejemplo, en el Estado de México y en Morelos el tipo Amarillo fue predominante, en Guerrero fue Rojo, en Oaxaca la mayoría de los agricultores poseen Ensaladillas, y en Tlaxcala fue el Beige.

El peso de 100 semillas varió de 22.4 a 78.1 g, que correspondieron al frijol 'Blanco enredador' (G137) de Guerrero y a la población Amarillo bola (E11) del Estado de México y Morelos, respectivamente (Cuadro 2). Solano-Cervantes et al. (2009) clasificaron los tamaños de grano de frijol de 


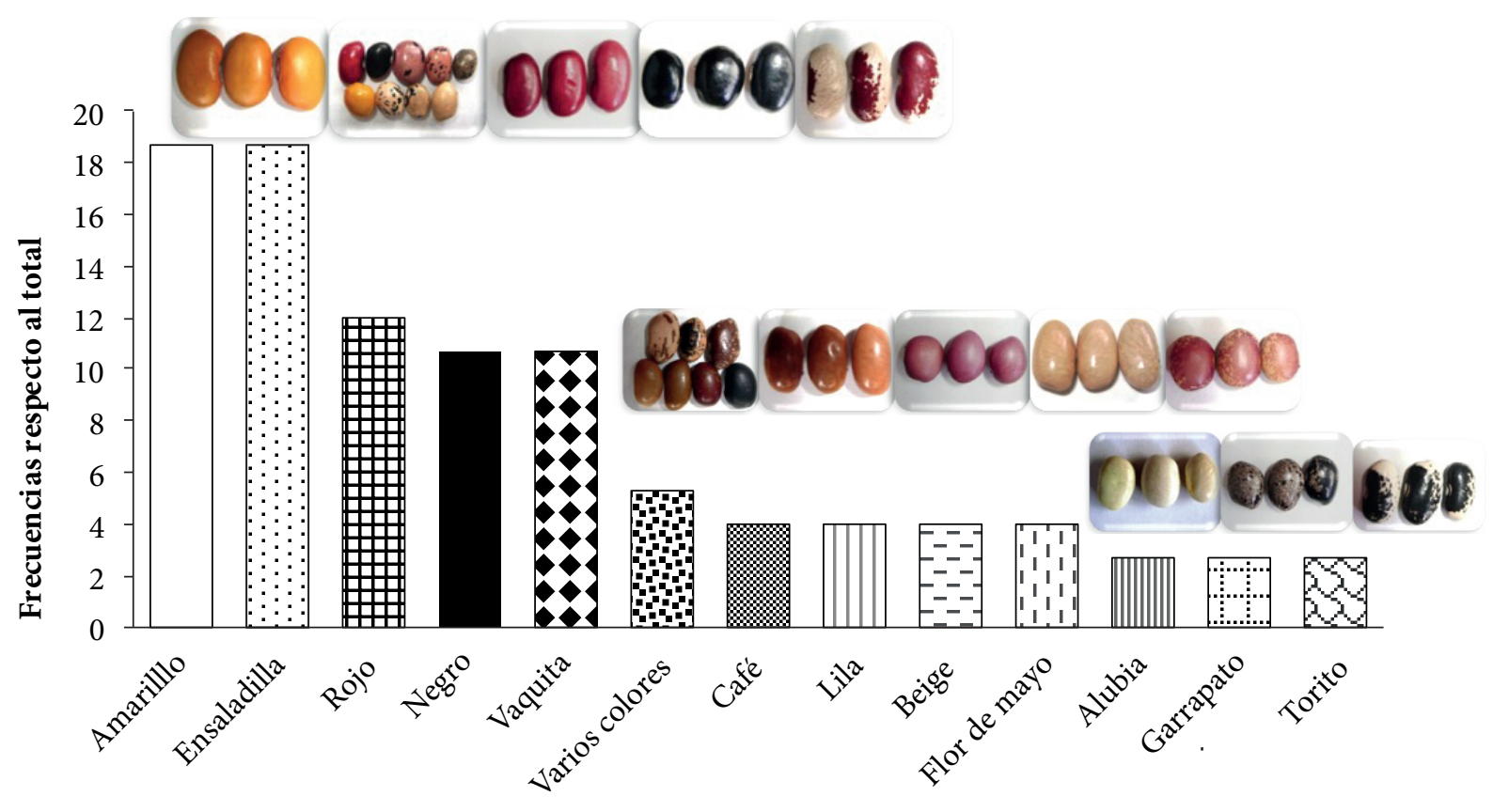

Color de semilla/ nombre local de frijol

Figura 1. Frecuencia de color o nombre de poblaciones de frijol trepador de los estados de México, Guerrero, Oaxaca y Tlaxcala $(n=75)$.

Guerrero, de acuerdo con la escala propuesta por Singh et al. (1991a, b) que consideran como de granos pequeños a los que pesan menos de $25 \mathrm{~g}$, medianos entre 25 a $40 \mathrm{~g}$, y grandes a los que superan $40 \mathrm{~g}$. De acuerdo con la forma y peso del grano, las poblaciones descritas corresponden de manera cercana a las razas Jalisco y Mesoamericana debido a que varían de forma oval, redondeada a arriñonada, cuyo peso oscila principalmente de 22.4 a $57.5 \mathrm{~g} / 100$ granos, $\mathrm{y}$ solo seis poblaciones superan $60 \mathrm{~g}$ (Figura 1, Cuadro 2). Los tamaños y pesos más bajos corresponden a poblaciones colectadas en Guerrero y Oaxaca, que fenotípicamente se asemejan más a la raza Mesoamericana (Singh et al., 1991a).

El volumen de 100 granos varió de $16.9 \mathrm{~cm}^{3}$ en frijol Blanco enredador (G137) de Guerrero a $66.4 \mathrm{~cm}^{3}$ en una variante de Amarillo bola (E11) del Estado de México. Con respecto a las dimensiones del grano, la longitud de semilla osciló de $0.97 \mathrm{~cm}$ en G129 (Rojo de vara) de Guerrero a $1.70 \mathrm{~cm}$ en E10 (Vaquita) del Estado de México. En ancho, la semilla de las poblaciones varió de $0.60 \mathrm{~cm}$ en G137 (frijol blanco) de Guerrero a $1.12 \mathrm{~cm}$ en un tipo Amarillo bola (E2) del Estado de México y de Morelos. El grosor de la semilla fluctuó entre $0.46 \mathrm{~cm}$ en la población E9 de Oaxaca conocido como frijol negro o negro-grueso, y $0.74 \mathrm{~cm}$ en una población de Amarillo del Estado de México y Morelos. La variación de tamaños y volúmenes de semilla confirman que en la región explorada se distribuyen las razas Mesoamericana y Jalisco, de acuerdo con la expresión fenotípica de dimensiones y forma de granos.

Los coeficientes de variación en características unidimensionales de semilla (longitud, anchura y grosor) son muy similares, entre 11.7 y $15.4 \%$; en tanto que las dimensiones de 100 granos, como peso y volumen, muestran una variación mayor ( $28 \%)$. Las diferentes dimensiones presentadas entre las poblaciones de frijol evaluadas corresponden a patrones geográficos de origen de las colectas, los que se distinguen a través de los análisis de componentes principales (Cuadro 2).

En el análisis de componentes principales (CP), los dos primeros explicaron $84.4 \%$ de la variación total. Las características de mayor importancia en la explicación del CP1 fueron longitud, anchura, peso y volumen de semilla, y en el CP2 fue grosor y peso específico de semilla, principalmente (Cuadro 3).

La distribución espacial de las poblaciones con base en los dos primeros componentes principales (Figura 2), mostró tres patrones fenotípicos definidos en función de las entidades federativas de origen de las poblaciones. Las poblaciones con dimensiones de semilla y peso específico mayor se encuentran en el cuadrante superior e inferior derecho (II y III) y corresponden principalmente a las originarias del Estado de México (E) y Morelos. Las provenientes de Oaxaca $(\mathrm{O})$ y Guerrero $(\mathrm{G})$ se ubican principalmente en el 
Cuadro 2. Variación de características físicas de la semilla de 75 poblaciones nativas de frijol de crecimiento indeterminado tipo IV.

\begin{tabular}{lcccccc}
\hline Descripción & Longitud $(\mathrm{cm})$ & Anchura $(\mathrm{cm})$ & Grosor $(\mathrm{cm})$ & $\begin{array}{c}\text { Peso de } 100 \\
\text { semillas }(\mathrm{g})\end{array}$ & $\begin{array}{c}\text { Volumen } 100 \\
\text { semillas }\left(\mathrm{cm}^{3}\right)\end{array}$ & $\begin{array}{c}\text { Peso específico } \\
\left(\mathrm{g} / \mathrm{cm}^{3}\right)\end{array}$ \\
\hline $\begin{array}{l}\text { Intervalo } \\
\begin{array}{l}\text { Desviación } \\
\text { estándar }\end{array}\end{array}$ & $0.973-1.703$ & $0.61-1.13$ & $0.47-0.75$ & $22.4-78.1$ & $16.9-66.4$ & $1.15-1.36$ \\
$\begin{array}{l}\text { Promedio } \\
\text { Coeficiente de }\end{array}$ & 0.19 & 0.13 & 0.07 & 12.5 & 10.7 & 0.15 \\
variación (\%) & 1.35 & 0.87 & 0.60 & 44.8 & 35.3 & 1.26 \\
\hline
\end{tabular}

Cuadro 3. Valores y vectores propios de los dos primeros componentes principales (CP) de la descripción de 75 poblaciones de frijol de crecimiento indeterminado tipo IV, con base en seis variables morfológicas.

\begin{tabular}{lcc}
\hline Característica evaluadas & $\mathrm{CP} 1$ & $\mathrm{CP} 2$ \\
\hline Longitud de semilla & 0.404 & 0.252 \\
Anchura de semilla & 0.484 & 0.007 \\
Grosor de semilla & 0.333 & -0.359 \\
Peso 100 semillas & 0.496 & 0.010 \\
Volumen 100 semillas & 0.491 & -0.099 \\
Peso específico & 0.064 & 0.893 \\
Valor propio & 3.977 & 1.047 \\
Variación explicada (\%) & 66.29 & 17.46 \\
Variación acumulada (\%) & 66.29 & 83.45 \\
\hline
\end{tabular}

cuadrante superior e inferior izquierdo (I y IV). Las poblaciones de Tlaxcala se dispersaron entre los cuadrantes I, II y IV. Esto es, las semillas de mayor tamaño corresponden esencialmente a las colectadas en el Estado de México y en Morelos, y las medianas y pequeñas fueron de Oaxaca y Guerrero. Esta diferencia entre las poblaciones del Estado de México en relación con las poblaciones de Guerrero y Oaxaca, se deben, en parte, a las formas de manejo agronómico y criterios de selección de semilla que hace el agricultor. En el primer caso se producen principalmente en monocultivo y algunas asociaciones, y en el segundo caso provienen del sistema milpa que conlleva la asociación de maíz-frijol o maíz-frijol-calabaza, entre otras combinaciones (Soleri et al., 2013).

La dispersión de los agrupamientos de las poblaciones por entidad federativa (Figura 2) confirma la correspondencia entre características morfológicas y orígenes de las poblaciones. En este sentido, Rocandio et al. (2009) determinaron patrones semejantes en 95 poblaciones de frijol común provenientes de Ozumba y Chalco, Estado de México en las que encontraron una amplia variación en forma, color y variegado de semilla, similar al presente estudio donde el frijol Amarillo y Ensaladilla fueron los más frecuentes.

Esos mismo patrones fueron determinados por Castillo et al. (2006) al evaluar la variación morfológica de $P$. vulgaris y $P$. coccineus en 100 poblaciones nativas de frijol en el oriente del Estado de México y norte de Morelos, cultivadas en asociación con maíz en el sistema milpa: en $P$. vulgaris encontraron la mayor variación en forma y color de semilla, de vaina y precocidad, y en $P$. coccineus la mayor variación en tamaño de semilla.

En el análisis de conglomerados (Figura 3) se muestra la formación de siete grupos a la distancia de 2.51 (pseudo $t$ = 57.1, $\mathrm{P}<0.05$ ). Las poblaciones E2, E11, E19, E29, E34 y E36 se separaron en un Grupo (I), el que se integró con poblaciones colectadas en el Estado de México y Morelos, que sobresalen porque poseen semillas grandes de $71.5 \mathrm{~g} / 100$ granos, 1.6 y $1.1 \mathrm{~cm}$ de longitud y anchura de semilla; y superaron a las poblaciones de frijol del Estado de México evaluadas por Castillo et al. (2006) que variaron de 14.9 a $57.3 \mathrm{~g} / 100$ granos.

El Grupo II está constituido por la población de grano pequeño originaria de Guerrero que no supera 23 g y 17 $\mathrm{cm}^{3}$ de volumen de 100 granos, denominada localmente como frijol Blanco enredador. El Grupo III está integrado por las poblaciones T5 de Tlaxcala y O40 de Oaxaca, de grano pequeño a mediano que no supera 36 g/100 granos, que corresponde a una combinación de frijol de testa negra, roja y pinto. El Grupo IV se integra por la población O46 que corresponde al frijol más pequeño de Oaxaca conocida como Cuarenteño o Negro delgado. El Grupo V se integró por un subgrupo de Oaxaca y otro de Guerrero de tamaño pequeño a mediano con un promedio en peso de $31.5 \mathrm{~g}$ y volumen de $25.3 \mathrm{~cm}^{3} / 100$ granos. Esto indica un probable flujo entre acervos silvestres, semicultivados y cultivados, como lo documentó Soleri et al. (2013) en Oaxaca y Zizumbo-Villarreal et al. (2005) en la región de Guanajuato y Michoacán. 


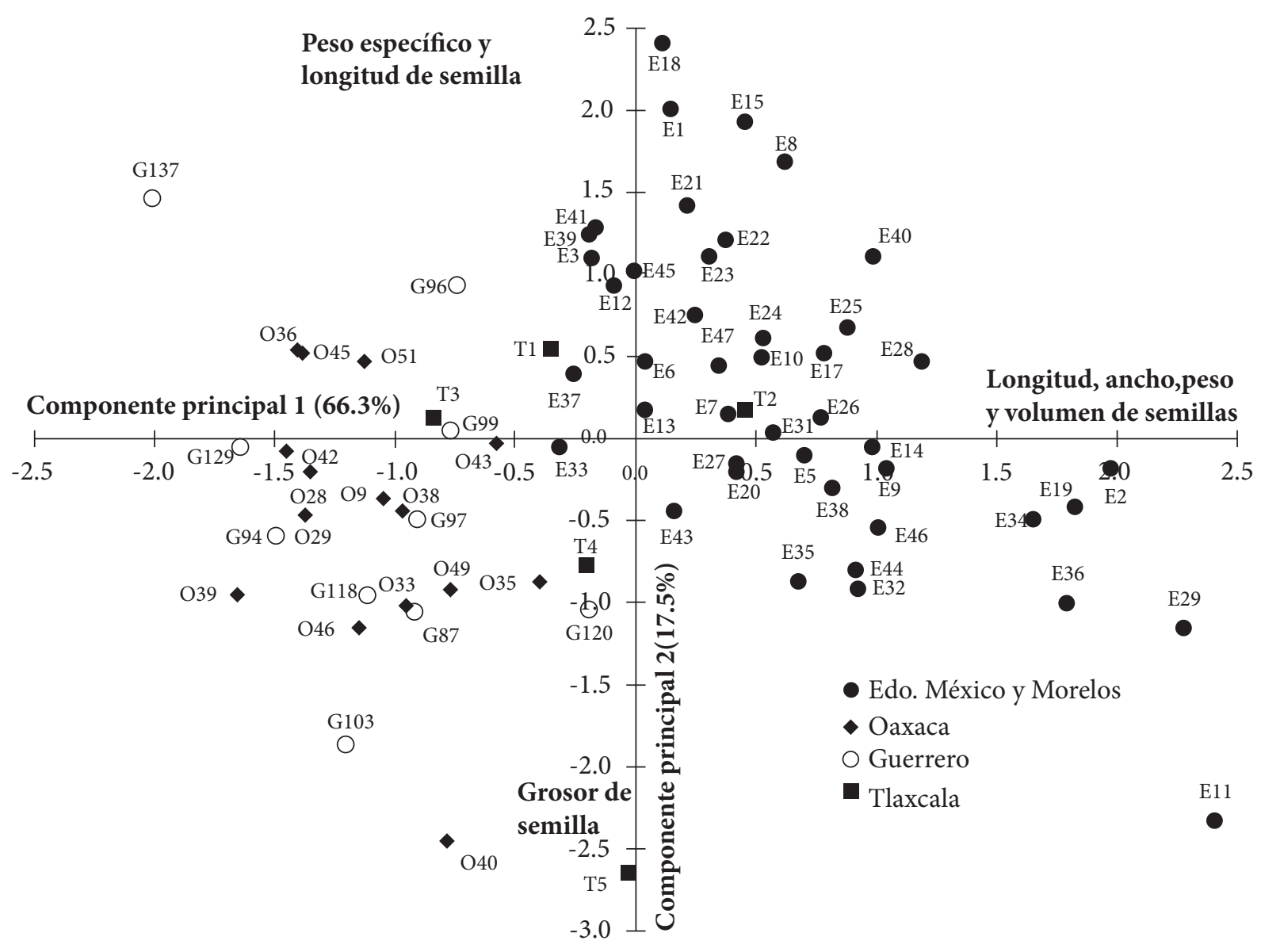

Figura 2. Distribución espacial de 75 poblaciones de frijol de hábito indeterminado tipo IV originarias de los estados de México y Morelos (E), Guerrero (G), Oaxaca (O) y Tlaxcala (T), con base en los dos primeros componentes principales de seis características morfológicas de semilla.

El Grupo VI está integrado por poblaciones colectadas en Oaxaca, Guerrero, Tlaxcala, Estado de México y Morelos; todas ellas con grano de tamaño mediano $(41.2 \mathrm{~g} / 100$ granos) y forma alargada $(1.4 \mathrm{~cm}$ de largo y $0.85 \mathrm{~cm}$ de ancho). El Grupo VII que incluye esencialmente a las poblaciones del Estado de México con tamaños medianos a grandes de 1.16 a $1.7 \mathrm{~cm}$ de longitud, 0.82 a $1.03 \mathrm{~cm}$ de ancho y peso de 38.3 a $60.5 \mathrm{~g}$. En este último grupo se observaron poblaciones con características relacionadas con las variedades comerciales Flor de Mayo, Cacahuate y Negro, entre otros. De acuerdo con la clasificación de Lépiz et al. (2010) sobre frijol de hábito trepador, las poblaciones aquí caracterizadas corresponden principalmente a formas intermedias a domesticadas cultivadas, que presentan más de $10 \mathrm{~g}$ de peso por 100 granos y longitudes superiores a 7.0 $\mathrm{mm}$, entre otros aspectos.

En cuanto a la relación entre características de semilla estimada mediante un análisis de conglomerados (Figura 4), se observó que el peso y volumen de 100 granos están estrechamente relacionados, seguido de anchura y longitud. El peso específico $\left(\mathrm{g} / \mathrm{cm}^{3}\right)$ y grosor de grano permitieron diferenciar las poblaciones de frijol, y el peso, anchura y longitud de semilla fueron las que determinaron las características diferenciales específicas entre poblaciones. Estos resultados permiten suponer que la diferenciación morfológica entre poblaciones y sus patrones de origen geográfico se pueden estimar mediante características de semilla, diferencias que posiblemente están asociadas con diferencias genéticas entre acervos regionales.

\section{CONCLUSIONES}

El recorrido, colecta y descripción de poblaciones de frijol de los estados de México, Morelos, Tlaxcala, Guerrero y Oaxaca, permitió definir 13 grupos de diversidad por color de grano y nombres locales. Se detectaron diferencias en morfología de semilla, entre poblaciones de diferente origen geográfico; las poblaciones del Estado de México difieren en características de semilla de las cultivadas en Oaxaca 


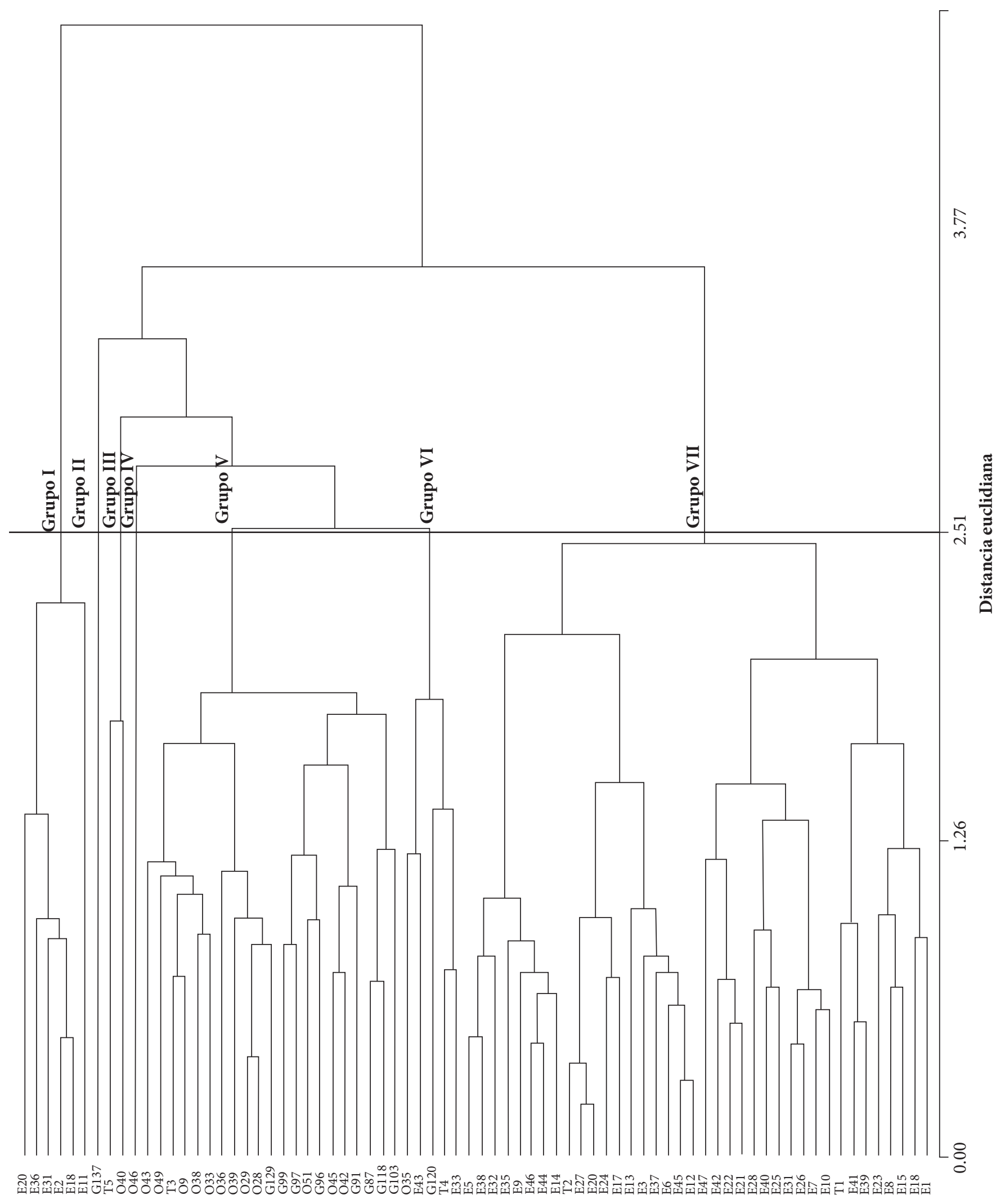

Figura 3. Dendrograma de 75 poblaciones de frijol de crecimiento indeterminado tipo IV, con base en el análisis de conglomerados de seis variables morfológicas de semilla. 


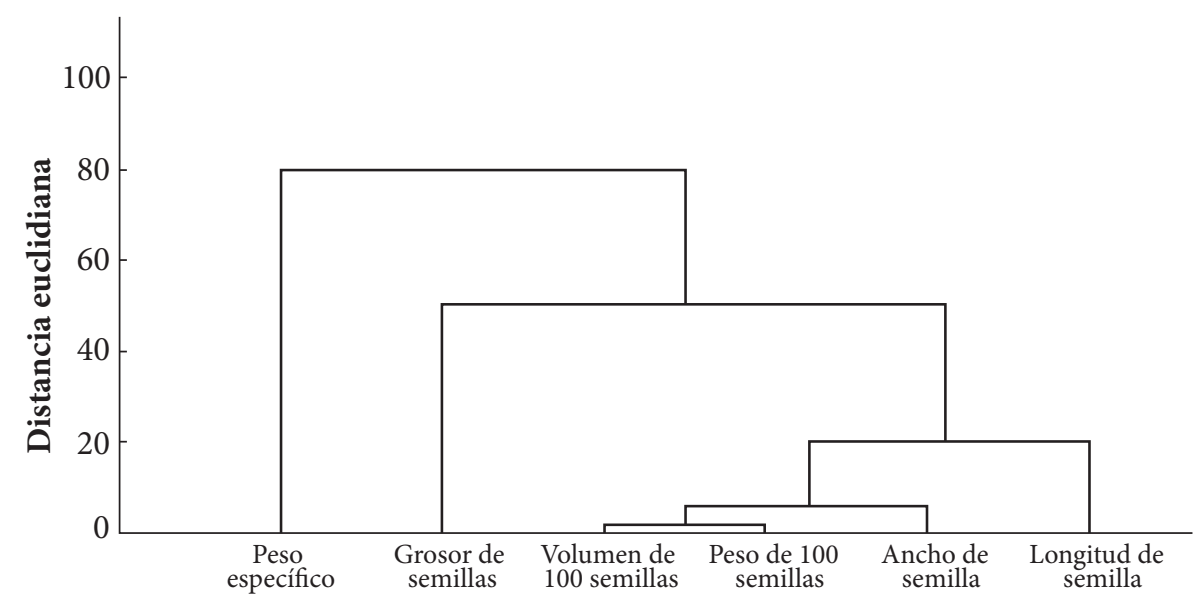

Figura 4. Relaciones de agrupamiento entre seis características morfológicas de semilla, con base en el análisis de 75 poblaciones de frijol de crecimiento indeterminado tipo IV.

y Guerrero. En el análisis de componentes principales, las variables de mayor valor discriminatorio entre poblaciones, fueron: longitud, ancho, peso y volumen de semillas y peso específico. En el análisis de conglomerados se determinaron siete grupos fenotípicos: dos grupos poblacionales del Estado de México y de Morelos, uno de semilla grande y otro de semilla mediana; dos grupos de semillas pequeñas, uno de Guerrero y otro de Oaxaca; y tres complejos grupales, uno con poblaciones de Tlaxcala y Oaxaca, otro con poblaciones de Oaxaca y Guerrero, y uno más que agrupó a poblaciones de Oaxaca, Guerrero, Estado de México, Morelos y Tlaxcala. Todos ellos difieren en tamaño y forma de semilla.

\section{AGRADECIMIENTOS}

Al CONACYT, Colegio de Postgraduados, Campus Montecillo, y SINAREFI por el financiamiento que hizo posible la realización de la presente investigación.

\section{BIBLIOGRAFÍA}

Aguilar J., C. Illsey y C. Marielle (2007) Los sistemas agrícolas de maíz y sus procesos técnicos. In: Sin Maíz no hay País. G. Esteva y C. Marielle (coord.). Consejo Nacional para la Cultura y las Artes. México, D.F. pp:83-122.

Bellon M. R. and J. Berthaud (2006) Traditional Mexican agricultural systems and the potential impacts of transgenic varieties on maize diversity. Agriculture and Human Values 23:3-14.

Bellon M. R., A. F. Barrientos-Priego, P. Colunga-GarcíaMarín, H. Perales, J. A. Reyes-Agüero, R. Rosales-Serna y D. ZizumboVillareal (2009) Diversidad y conservación de recursos genéticos en plantas cultivadas. In: Capital Natural de México, Vol. II: Estado de Conservación y Tendencias de Cambio. R. Dirzo, R. González y I. J. March (comps.). CONABIO, México, D.F. pp:355-382.

Boege E. (2010) El Patrimonio Biocultural de los Pueblos Indígenas de México. Hacia la Conservación in situ de la Biodiversidad y Agrobiodiversidad de los Territorios Indígenas. Instituto $\mathrm{Na}$ - cional de Antropología e Historia y Comisión Nacional para el Desarrollo de los Pueblos Indígenas, México, D.F. 342 p.

Castillo M., P. Ramírez, F. Castillo y S. Miranda (2006) Diversidad morfológica de poblaciones nativas de frijol común y frijol ayocote del oriente del Estado de México. Revista Fitotecnia Mexicana 29:111-119.

De Allende G., M. G. Rivera, R. Rosales, M. G. Acero y N. Mayek (2006) Calidad bioquímica del frijol cultivado en distintas condiciones de humedad del suelo. Investigación y Ciencia 34:12-18.

Di-Rienzo J. A., F. Casanoves, M. G. Balzarini, L. González, M. Tablada y C. W. Robledo (2008) InfoStat Software Estadístico Versión 2008. Grupo InfoStat, FCA-Universidad Nacional de Córdoba. Córdoba, Argentina.

Hernández-López V. M., M. L. Vargas-Vázquez, J. S. Muruaga-Martínez, S. Hernández-Delgado y N. Mayek-Pérez (2013) Origen, diversidad y diversificación del frijol común. Avances y perspectivas. Revista Fitotecnia Mexicana 36:95-104.

Lépiz R., J. J. López, J. J. Sánchez, F. Santacruz, R. Nuño y E. Rodríguez (2010) Características morfológicas de formas cultivadas, silvestres e intermedias de frijol común de hábito trepador. Revista Fitotecnia Mexicana 33:21-28.

López J. L., J. A. Ruiz, J. J. Sánchez y R. Lépiz (2005) Adaptación climática de 25 especies de frijol silvestre (Phaseolus spp.) en la República Mexicana. Revista Fitotecnia Mexicana 28:221-230.

Nadal A. (2000) The environmental and social impact of economic liberation on corn production in Mexico: a study commissioned by Oxfam GB and WWF International. WWF and Oxfam GB Gland, Switzerland, $130 \mathrm{p}$.

Pinedo R., L. Collado, L. Arias y T. Shagarodsky (2009) Importancia del maíz, frijol, pallar y chile en agroecosistemas tradicionales del trópico húmedo de Cuba, México y Perú. In: ¿Cómo Conservan los Agricultores sus Semillas en el Trópico Húmedo de Cuba, México y Perú? Experiencias de un Proyecto de Investigación en Sistemas Informales de Semillas de Chile, Frijoles y Maíz. M. Hermann, K. Amaya, L. Latournerie y L. Castiñeiras (eds.). Bioversity International, Roma, Italia. pp:31-45.

Rocandio M., P. Ramírez, F. Castillo, S. Miranda y J. A. Gómez (2009) Diversidad en características de interés agronómico de poblaciones nativas de frijol común en asociación con maíz. In: Memoria del X Simposio Internacional y V Congreso Anual de Agricultura Sostenible. Sociedad Mexicana de Agricultura Sostenible, A.C. (ed.). 9-14 de noviembre. Chiapas, México. pp:44-52.

Romero-Arenas O., M. A. Damián-Huato, J. A. Rivera-Tapia, A. BáezSimón, M. Huerta-Lara and E. Cabrera-Huerta (2013) The nutritional value of beans (Phaseolus vulgaris L.) and its importance for feeding of rural communities in Puebla, Mexico. 
International Research Journal of Biological Sciences 2:59-65.

Salinas R. A., J. A. Acosta, E. López, C. Torres, F. J. Ibarra y R. Félix (2008) Rendimiento y características morfológicas relacionadas con tipo de planta erecta en frijol para riego. Revista Fitotecnia Mexicana 31:203-211.

SAS, Statistical Analysis System (2002) SAS System for Windows Version 9.0 by SAS Institute Inc. Cary, NC, USA.

Singh S. P., P. Gepts and D. G. Debouck (1991a) Races of common bean (Phaseolus vulgaris, Fabaceae). Economic Botany 45:379-396.

Singh S. P., J. A. Gutierrez, A. Molina, C. Urea and P. Gepts (1991b) Genetic diversity in cultivated common bean: II. Marker based analysis of morphological and agronomic traits. Crop Science 31:23-29.

Solano-Cervantes F., R. Díaz-Ruiz, C. Jacinto-Hernández, L. AguirreÁlvarez y A. Huerta-de-la-Peña (2009) Prácticas agrícolas, descripción morfológica, proteínica y culinaria del grano de cultivares de frijol sembrados en la región de Tlatzala, Guerrero. Ra Ximhai 5:187-199.

Soleri D., M. Worthington, F. Aragón-Cuevas, S. E. Smith and P. Gepts (2013) Farmers' varietal identification in a reference sample of local Phaseolus species in the Sierra Juárez, Oaxaca, México. Economic Botany 67:283-298.

Vargas-Vázquez M. L. P., J. S. Muruaga-Martínez, P. Pérez-Herrera, H. R. Gill-Langarica, G. Esquivel-Esquivel, M. A. MartínezDamián, R. Rosales-Serna y N. Mayek-Pérez (2008) Caracterización morfoagronómica de la colección núcleo de la forma cultivada de frijol común del INIFAP. Agrociencia 42:787-797.

Vidal-Barahona A., L. C. Lagunes-Espinoza, E. Valadez y C. F. OrtizGarcía (2006) Variabilidad morfológica y molecular de cultivares criollos y mejorados de frijol común en Tabasco, México. Revista Fitotecnia Mexicana 29:273-281.

Vizcarra I. y N. Marín (2006) Las niñas a la casa y los niños a la milpa: la construcción social de la infancia mazahua. Convergencia 40:39-67.

Zizumbo-Villarreal D., P. Colunga-GarciaMarín, E. Payró-de-la-Cruz, P Delgado-Valerio and P. Gepts (2005) Population structure and evolutionary dynamics of wild-weedy-domesticated complexes of common bean in a Mesoamerican region. Crop Science 45:1073-1083. 